\title{
Tracking oscillations in the presence of delay-induced essential instability
}

\author{
J. Sieber ${ }^{a, *}$ and B. Krauskopf ${ }^{b}$ \\ ${ }^{a}$ Centre for Applied Dynamics Research, School of Engineering, University of \\ Aberdeen, Fraser Noble Building, King's College, Aberdeen AB24 3UE, United \\ Kingdom \\ b Department of Engineering Mathematics, University of Bristol, Queen's \\ Building, Bristol BS8 1TR, United Kingdom
}

\begin{abstract}
Hybrid testing, which couples physical experiments and computer simulations bidirectionally and in real-time, is a promising experimental technique in engineering. One fundamental problem of this technique are delays in the coupling between simulation and experiment. We discuss this issue for a simple prototype hybrid system: a pendulum that is vertically excited by coupling it to a simulated linear massspring-damper system. Under realistic conditions a small delay in the coupling can give rise to an essential instability: the linearisation has infinitely many unstable eigenvalues for arbitrarily small delay. This type of instability is impossible to compensate for with any of the standard compensation techniques. We introduce an approach based on feedback control and Newton iterations that is able to overcome this instability. The basic idea behind our approach consists of two parts. First, we change the bidirectional coupling between experiment and computer simulation to a unidirectional coupling and stabilise the experiment with a feedback loop. Second, we place the modified hybrid system into a Newton iteration scheme. If the iteration converges then the hybrid experiment behaves just as the original emulated system (within the experimental accuracy). We demonstrate, by using a computer simulation for the experimental part, that our approach is able to overcome the essential instability. In combination with path-following methods, it allows us to track oscillations and their bifurcations systematically.
\end{abstract}

Key words: hybrid testing, coupling delay, bifurcation analysis.

\footnotetext{
* Corresponding author.

Email addresses: J.Sieber@abdn.ac.uk (J. Sieber),

B.Krauskopf@bristol.ac.uk (and B. Krauskopf).
} 


\section{Introduction}

Nonlinear oscillations are a well-known feature of nonlinear dynamical systems. When a mathematical model of the system under consideration is known, for example, in the form of an ordinary differential equation (ODE), then these nonlinear oscillations can be tracked, regardless of their dynamical stability, in a suitable parameter as periodic solutions of the ODE. Furthermore, their bifurcations (stability boundaries, for example, period doublings) can be detected and tracked in two or more parameters. This tracking (one also speaks of path-following or numerical continuation) of different types of solutions and their bifurcations has emerged as a tremendously useful tool for the analysis of nonlinear dynamical systems. It can be performed with a number of freely available software packages; see, for example, the recent survey [3].

In this paper we consider the problem of tracking oscillations and stability boundaries directly in an experiment, that is, in a situation when a full mathematical model of the system under consideration is not available. More specifically, we are interested in hybrid testing experiments and, in particular, in real-time dynamic substructured testing of mechanical and civil engineering systems [4-7]. This term refers to the splitting of a complex structure into two components (in the simplest case). The first component, for which a reliable model is available, is simulated on the computer. The second component, typically the part that contains nonlinearities that are difficult to model reliably, is coupled bidirectionally and in real time to the computer model of the first component. The coupling in one direction is done by a transfer system (for example, a servo-mechanical actuator) that enforces the displacements computed in the numerical simulation onto the experimental component. To close the loop, the forces measured at the actuator are fed into the numerical simulation, where they enter as an inhomogeneity.

Section 2 explains the general hybrid setup by using a concrete example: a substructured version of a nonlinear vibration damper in the form of a real pendulum that is coupled at its pivot to a computer simulation of a vertically excited mass-spring-damper (MSD) system; see Fig. 1. Throughout the paper we formulate all statements and algorithms for this prototype nonlinear

hybrid experiment. Note that the original emulated pendulum-MSD system (a periodically driven two-degree of freedom oscillator) can be modelled easily and shows a rich bifurcation structure, which makes the pendulum-MSD system an ideal test candidate for experimental bifurcation analysis.

One major issue in hybrid testing is the presence of unavoidable delays between the experimental part and the computer model, which may lead to an instability of the coupled system. In many situations delay compensation techniques are able to overcome this problem [5,7]. However, as we demonstrate 


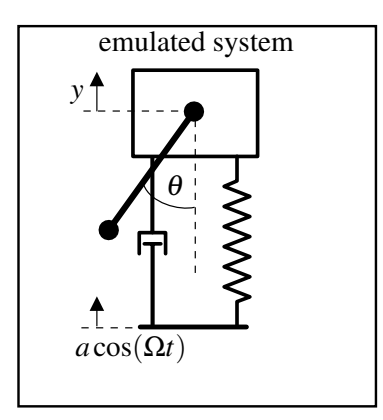

(a)

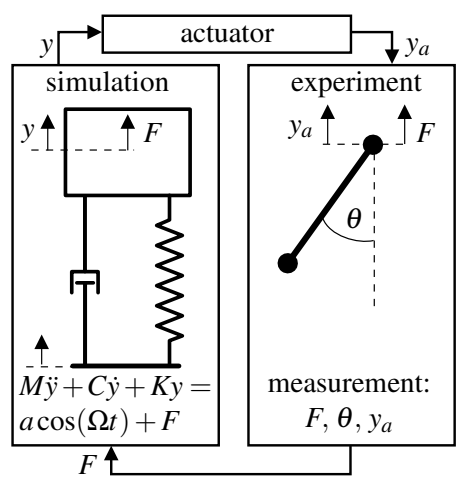

(b)

Fig. 1. Sketch of the decomposition of the overall pendulum-MSD system into a computer simulation of a mass-spring-damper system and a real pendulum. Panel (a) shows the original (emulated) system, and panel (b) the bidirectionally real-time coupled system as studied in [6,7], where the pendulum is of mass $m=0.27 \mathrm{~kg}$ and length $l=0.1955 \mathrm{~m}$.

in Section 3, for certain hybrid tests delays give rise to an essential instability, meaning that at the linear level the delay coupled system has infinitely many unstable eigenvalues regardless of the size of the delay. Section 3 and Section 4 show for the hybrid pendulum-MSD system how the instability arises and why it persists even when one applies delay compensation.

The centrepiece of our paper is Section 5 where we show how the delay-induced essential instability can be overcome in the context of bifurcation analysis for experiments $[8,9]$. The basic idea can be explained for the pendulum-MSD system as follows; for the mathematical formulation see Section 5.

- We decouple the actuator from the computer simulation and instead drive the pendulum (the experimental part, in general) with a periodic signal. In other words, the experimental part is periodically driven and it feeds the force measurement back into the computer simulation (see already Fig. 2).

- We add a stabilising feedback loop with a periodic control demand to the experimental component. In the pendulum-MSD example this should be a proportional-plus-derivative (PD) control that depends on the measured angle $\theta(t)$ of the pendulum. This type of $\mathrm{PD}$ control can be achieved, for example, by applying a torque at the pivot point.

The decoupled hybrid system produces stable periodic output in reaction to the periodic input. In other words, whatever periodic input we choose, the pendulum will settle to a periodic state of the same period after a short transient. This provides us with an output in the form of the motion $y_{a}(t)$ of the actuator, the motion $\theta(t)$ of the angle, and the computed displacement output $y(t)$ of the simulation.

The key realisation is that the overall controlled hybrid system reproduces the original emulated system perfectly when the control signal is zero and 
the actual motion $y_{a}(t)$ agrees with the computed motion $y(t)$ (within the experimental accuracy). This means that experiment and simulation are synchronised and the feedback control is non-invasive.

- The conditions of non-invasiveness and synchronisation can be formulated as a nonlinear root finding problem. Specifically, the inputs and the outputs are periodic time profiles with the same period (the period of the input). Even though there is, in general, no explicit formula for the input-output dependence, we know that it is smooth. This enables us to find periodic inputs that correspond to synchronised motion with non-invasive control by means of a Newton iteration.

- Finally, we embed the Newton iteration into a path-following procedure, which means that we track how the solution changes depending on one system parameter. Here the system parameter is changed only slightly, which ensures that the Newton method convergences at the new parameter value. Furthermore, it is then also possible to formulate and add bifurcation conditions to track bifurcations in two or more system parameters.

We refer to the above approach as control-based bifurcation analysis. It does not require an underlying model of the overall dynamical system but instead it relies on feedback stabilizability of the experimental component, which is generally a mild assumption in the context of a hybrid test. Moreover, we do not need to set the initial conditions for the experimental component, which would be difficult in practice. The Newton iteration and the path-following do not have to be performed in real time. Similarly, the simulation of the numerical model does not have to be performed in real time anymore. Only the feedback loop of the experimental part has to meet real-time requirements. We remark that the overall test captures nevertheless the dynamics of the original emulated system accurately: it produces exact results when experiment and simulation are synchronised. Importantly, the problem of delay-induced essential instability of the hybrid system disappears within the framework of control-based bifurcation analysis, because dynamically the system is always only coupled in one direction.

Section 6 presents a feasibility study of the above approach for the pendulumMSD system, where we use a computer simulation of the pendulum with limited accuracy to track the stability boundary of the hanging-down state, as well as a family of periodic solutions. Control-based bifurcation analysis of an actual hybrid pendulum-MSD experiment is work in progress, because it requires the addition of a feedback (torque) control loop to the experimental setup from $[6,7]$. 


\section{Pendulum-MSD system as a prototype hybrid experiment}

Hybrid test experiments - also called substructured experiments - have originally been developed to test large structures in civil engineering. They couple a critical, nonlinear or poorly understood component of the overall structure to a real-time simulation of a numerical model of the remainder of the structure [4,10]. This critical component can be, for example, a support cable of a bridge, or a damper that keeps helicopter blades apart. The other main motivation for substructuring a test is that it opens the opportunity for systematic parameter studies. If one replaces a well-modelled part of the experiment with a simulation then system parameters of this part (for example, masses or stiffnesses) can be varied easily without physically changing, or even stopping, the experiment; see [7] for a prototype study.

We consider here as the emulated system a pendulum that is attached to a linear vertically excited mass-spring-damper (MSD) system; see Fig. 1(a). The pendulum-MSD system is a periodically driven oscillator with a geometric nonlinearity and two degrees of freedom. It is a prototype system for autoparametric resonance phenomena as they occur, for example, in bridge cables [6]. The pendulum-MSD system also acts as a nonlinear vibration damper for the vertical motion of the mass. Throughout the paper we will formulate all statements and algorithms for the pendulum-MSD example, which can be modelled as

$$
\begin{aligned}
M \ddot{y}+C \dot{y}+K y & =a \cos (\Omega t)-m \ddot{y}-m l\left[\ddot{\theta} \sin \theta+\dot{\theta}^{2} \cos \theta\right], \\
\ddot{\theta}+\frac{\kappa}{m l^{2}} \dot{\theta}+\left[\frac{g+\ddot{y}}{l}\right] \sin \theta & =0 .
\end{aligned}
$$

Here $\theta$ is the angular displacement of the pendulum, $y$ is the vertical displacement of the pendulum pivot that is attached to the mass, $M, C$ and $K$ are the mass, damping and stiffness of the MSD system, $m$ is the mass of the pendulum, $l$ is the length of the pendulum, $g$ is the acceleration due to gravity, and $a$ and $\Omega$ are the amplitude and the frequency of the forcing. A force amplitude of $a$ corresponds to a displacement excitation of amplitude $a / \sqrt{\Omega^{2} C^{2}+K^{2}}$ of the MSD.

The pendulum-MSD system has a rich but well understood bifurcation structure. For the mathematical model (1)-(2) all bifurcations of periodic orbits (period doubling, symmetry breaking, torus and saddle-node bifurcations, homoclinic and heteroclinic tangencies) can be explored systematically with numerical continuation methods as implemented in AUTO [11]; see also [2,12] for more background information on numerical continuation.

A typical setup of a hybrid experiment is sketched in Fig. 1(b), where the pendulum-MSD system is decomposed into a simulation and a real experiment. 
In this setup, which was realised in [6,7], the mechanical part of the hybrid experiment is a pendulum of mass $m=0.27 \mathrm{~kg}$ and effective length $l=0.1955 \mathrm{~m}$. The linear viscous friction coefficient $\kappa$ is estimated as $7.5 \cdot 10^{-3} \mathrm{~kg} / \mathrm{s}$ from the results in [7]. The pendulum is attached at its pivot to a transfer system, which is a servo-mechanical actuator in the experiment in [7]. The transfer system is provided with a trajectory $y(t)$ for its vertical motion, which is calculated by numerical simulation of the linear MSD system

$$
M \ddot{y}+C \dot{y}+K y=a \cos (\Omega t)+F(t) .
$$

The numerical model (3) has an inhomogeneity $F(t)$ that originates from force measurements at the pivot of the pendulum. This means that the hybrid experiment involves a loop between three stages as schematically shown in Fig. 1(b):

- the numerical simulation of model (3) with the force input $F(t)$;

- feeding the actuator with the simulation output $y(t)$, and

- feeding back the force measurement $F(t)$ to the simulation.

All parts of the loop need to be implemented in real time, and need to run simultaneously and in parallel to the experiment. One advantage of the hybrid setup is that one can easily and systematically vary the system parameters of the numerical subsystem ( $a, \Omega, M, C$ and $K$ in our case), while testing the nonlinear structure (the pendulum) experimentally and in its original size.

\section{Essential instability due to delay in the coupling}

The real-time coupling between simulation and experiment via a transfer system and force measurements introduces a number of difficulties. Apart from the inherent noisiness of the force measurements, the most severe problem is the mismatch between the prescribed trajectory $y(t)$ obtained from the simulation and the output $y_{a}(t)$ of the transfer system; see Fig. 1(b). This mismatch

$$
e(t)=y_{a}(t)-y(t)
$$

is called the synchronisation error. It is caused by the dynamics of the actuator, which is in general not able to follow the prescribed input trajectory perfectly and instantly. Since the output of the simulation $y(t)$ is known, the synchronisation error (4) can be determined in a hybrid experiment by recording the actual motion $y_{a}(t)$ of the transfer system (the mechanical actuator). Generally, the smallness of the synchronisation error $e(t)$ is taken as a measure of accuracy of the whole hybrid experiment $[5,13]$.

In many situations the actual trajectory $y_{a}(t)$ of the actuator follows the pre- 
scribed trajectory $y(t)$ almost exactly, but with a fixed small pure time delay $\tau[6,13,14]$. Hence, the actuator can be modelled as

$$
y_{a}(t)=y(t-\tau) .
$$

Inserting this actuator model (5) into the mathematical model of the combined system leads to a mathematical model in the form of a delay differential equation (DDE). Two facts support the modelling of the actuator by a pure delay. First, the DDE model predicts an instability of the hybrid experiment for delays larger than a certain critical delay $\tau_{c}$ for many cases: for example, in the case $m<M$ for the pendulum-MSD; see [6]. The experimental observations confirmed this prediction of instability and even showed a precise quantitative agreement with the predicted values of the critical delays $\tau_{c}$ and the predicted frequency of the growing vibration. This type of agreement was demonstrated not only for the pendulum-MSD system but also for other prototype experiments for which models are available, such as other multi-MSD hybrid experiments $[6,13,15]$. Second, the delay compensation techniques developed on the basis of the idealised model $(5)$ in $[5,14,16]$ have been successful in suppressing these instabilities and reducing the synchronisation error $e(t)$ significantly, for example, in the studies [5,7]. One underlying assumption behind the actuator model (5) is that the modulus of the force $F(t)$ is moderate, meaning that it has a negligible influence on the dynamics of the actuator.

Specifically for the pendulum-MSD system considered here, inserting (5) into (1) $-(2)$ gives (see [6])

$$
\begin{aligned}
M \ddot{y}+m l \sin \theta \ddot{\theta}+m \ddot{y}(t-\tau)+C \dot{y}+K y+m l \dot{\theta}^{2} \cos \theta & =a \cos (\Omega t), \\
\ddot{\theta}+\frac{\kappa}{m l^{2}} \dot{\theta}+\frac{1}{l}[g+\ddot{y}(t-\tau)] \sin \theta & =0,
\end{aligned}
$$

where we have dropped the argument $t$ for all dependent variables, except for those that feature the delay $\tau$. The fact that system (6) has terms that depend on the state some time $\tau$ ago has some important consequences. The state space of (6) is infinite dimensional: the evolution depends on the history of $\dot{y}$ in the time interval $[t-\tau, t]$. What is more, the delay enters in the highest derivative $\ddot{y}(t-\tau)$ of $y$, which means that (6) is an example of a neutral delay differential equation [17].

This neutrality is the reason why system (6) shows an extreme instability if $m>M$ near angles $\theta=0$ or $\pi$ for arbitrarily small delay $\tau$. Deriving this result is quite technical in nature and details can be found in Appendix A. The key step is to bring system (6) into the form of an explicit first-order system consisting of a difference equation and an ODE; see the textbooks $[17,18]$. This first-order formulation (system (A.1)-(A.5) in Appendix A) of (6) allows us to clarify in which sense the system is a well-posed initial-value problem for a dynamical system, and how one can determine the stability properties 
of linearisations along trajectories of (6). Specifically, the theory developed in [17] then implies that the time map (say, from some time $t_{1}$ to $t_{1}+\delta$ where $\delta>0$ ) of the relevant linearisation has (countably) infinitely many eigenvalues $\lambda_{j}(j=-\infty, \ldots, \infty)$ and that

$$
\left|\lambda_{j}\right| \rightarrow \rho \text { for }|j| \rightarrow \infty
$$

where $\rho$ is the essential spectral radius of the time map of the linear difference equation

$$
u(t)=\left[-\frac{m}{M} \cos ^{2} \theta_{*}(t)\right] u(t-\tau) .
$$

Here $\theta^{*}(t)$ is the angle of the solution around which we linearise, and $u$ is the linear variation of $\dot{y}$; see Appendix A for details. If $m>M, \theta_{*}\left(t_{1}\right)=0$, and $\delta>0$ is sufficiently small then the prefactor of $u(t-\tau)$ in (8) satisfies

$$
\left|-\frac{m}{M} \cos ^{2} \theta_{*}(t)\right|>\rho_{0}
$$

for some $\rho_{0}>1$. Thus, the essential spectral radius $\rho$ of the time map from $t_{1}$ to $t_{1}+\delta$ for the difference equation (8) satisfies

$$
\rho \geq\left(\rho_{0}\right)^{\delta / \tau}
$$

This means that the right-hand-side of estimate (10) is larger than 1 for arbitrarily small delays $\tau$. Even more, it grows strongly for $\tau \rightarrow 0$. Equation (7) implies that, at least for time $\delta$, the linearisation along any trajectory of the coupled system (6) passing through $\theta=0$ (or $\theta=\pi$ ) has infinitely many eigenvalues with modulus greater or equal to $\left(\rho_{0}\right)^{\delta / \tau}$, which is dramatically larger than 1 for $m>M$. Consequently, any small disturbance occurring in the hybrid experiment will be amplified to order 1 whenever $\theta$ is close to 0 or $\pi$. The fact that infinitely many eigenvalues and the essential spectral radius of the linearisation are larger than 1 motivates our notion of referring to this case as an essential instability.

The frequencies corresponding to the unstable eigenvalues have (asymptotically) a spacing of $2 \pi / \tau$. Thus, most of the unstable eigenvalues have large frequencies (imaginary parts). A real mechanical actuator is not capable of supporting an instability at infinitely many frequencies. Typically, the actuator will have an internal control loop that guarantees that the actuator dynamics is a stiff approximation of the idealisation (5), for example,

$$
m_{a} \ddot{y}_{a}+c_{a} \dot{y}_{a}+\omega_{a}^{2}\left[y_{a}-y(t-\tau)\right]=F
$$

for large positive $\omega_{a}$ and $c_{a}$, where $F$ is the force measured at the pivot and $m_{a}$ is the mass of the actuator. This gives rise to a regularisation of the ill-posed problem (8), which nevertheless has a large number of strongly unstable eigenvalues for large $\omega_{a}$ and $c_{a}$, and small delays. Hence, the essential instability 
renders the hybrid test practically infeasible for $m>M$ also for a real actuator rather than an idealised one. Paradoxically, the results of this section imply that the instability becomes more dramatic if we "improve" the actuator by decreasing the delay and increasing $\omega_{a}$ and $c_{a}$.

\section{Failure of classical delay compensation}

The delay compensation methods developed in $[5,14]$ and applied in $[5,7]$ are not able to overcome the essential instability discussed in Section 3. The choice of delay compensation methods for hybrid experiments is more restricted than in the classical field of delay compensation for feedback control where many more approaches have been proved to work; see, for example, [20-22]. The reason behind this restriction is that the hybrid system with compensation not only has to be stable, but it also needs to approximate the unknown dynamics of the emulated system - namely (1)-(2) in our case.

The two methods developed in $[5,14]$ are based on polynomial extrapolation. This means that the input for the actuator is not $y(t)$ but $P[y](t+\hat{\tau})$ where $P[y]$ is an interpolation polynomial obtained from a history segment of $y$, and $\hat{\tau}$ is an estimate of the delay time. In [5] $\hat{\tau}$ is adapted along the trajectory and the polynomial $P$ is the second- or fourth-order least-squares fitting polynomial of $\left(y\left(t-k \tau_{s}\right), \ldots, y\left(t-\tau_{s}\right), y(t)\right)$ where $\tau_{s}$ is the step size of the numerical simulation (which is equal to the sampling time of the experiment). Thus, in the actuator model $(5)$ the term $y(t-\tau)$ is replaced by $P[y(\cdot-\tau)](t+\hat{\tau})$. Because the evaluated least-squares fitting polynomial is a linear combination of the interpolated values of $y$, the term $y(t-\tau)$ in (6) is effectively replaced with a linear combination of past terms of $y$ of the form

$$
P[y(\cdot-\tau)](t+\hat{\tau})=c_{0} y(t-\tau)+\ldots c_{k} y\left(t-\tau-k \tau_{s}\right) .
$$

One condition on the polynomial extrapolation (in fact, of any delay compensation scheme) is consistency, which means that

$$
c_{0}+\ldots+c_{k}=1 \text {. }
$$

Only the consistency condition (13) guarantees that the compensation is accurate at least for $\hat{\tau}=\tau=0$. Inserting the compensation (12) instead of $y(t-\tau)$ into system (6), which models the hybrid pendulum-MSD system, we obtain a neutral delay differential equation that has the difference equation

$$
u(t)=\left[-\frac{m}{M} \cos ^{2} \theta_{*}(t)\right]\left[c_{0} u(t-\tau)+\ldots c_{k} u\left(t-\tau-k \tau_{s}\right)\right]
$$

as its essential part instead of (8). In (14) the coefficients $c_{0}, \ldots, c_{k}$ satisfy $c_{0}+\ldots+c_{k}=1$. Instead of the single small delay $\tau$ in the essential part (8) 
the difference equation (14) has multiple delays, $\tau$ up to $\tau+k \tau_{s}$. Typically, all of these delays are small $\left(\tau \sim 10 \mathrm{~ms}, \tau_{s}=1 \mathrm{~ms}\right)$. Computing the essential spectral radius of the time map from $t_{1}$ to $t_{1}+\delta$ for (14) analytically is difficult. However, the theory of neutral equations [17] states that for any difference equation of the form

$$
u(t)=d_{0} u\left(t-\tau_{0}\right)+\ldots+d_{k} u\left(t-\tau_{k}\right) \quad \text { where }\left|d_{0}\right|+\ldots+\left|d_{k}\right|>\rho_{0}
$$

there exists a $(k+1)$-tuple of delays $\left[\tilde{\tau}_{0}, \ldots, \tilde{\tau}_{k}\right]$ arbitrarily close to $\left[\tau_{0}, \ldots, \tau_{k}\right]$ such that the essential spectral radius of the time- $\delta$ map of

$$
u(t)=d_{0} u\left(t-\tilde{\tau}_{0}\right)+\ldots+d_{k} u\left(t-\tilde{\tau}_{k}\right)
$$

is larger than $\left(\rho_{0}\right)^{\delta / \tau_{k}}$. This also holds for time-dependent coefficients $d_{0}, \ldots, d_{k}$. For a consistent delay compensation scheme this implies that the scheme is uncontrollably sensitive with respect to the delays $\tau, \ldots, \tau+k \tau_{s}$ whenever the hybrid system (6) is essentially unstable in our sense, that is, (for the pendulum-MSD system) whenever condition (9) is satisfied. In other words, any delay compensation scheme applied to a system with an essential instability will also have an essential instability.

In the interpretation of the pendulum as a nonlinear damper of the MSD system, the condition $m>M$ means that the mass of the damper is larger than that of the damped system. While the emulated system does not display any special properties in this case, our mathematical analysis shows that the hybrid experiment develops an extreme sensitivity to delays. A physical reason behind this somewhat surprising result lies in the fact that the two subsystems are coupled at a fixed joint (in contrast to a spring), while one is prescribing displacements and measuring forces at the interface; see Fig. 1 (b). If $m<M$ (or the coupling is via a spring) then instabilities can still occur but they involve only a small number of eigenvalues when the delay is small (typically $\approx$ $10 \mathrm{~ms}$ ). Hence, classical delay compensation is suitable for these non-essential instabilities [5]. However, as we have seen, for $m>M$ delay compensation fails and it is impossible to achieve an approximation of the dynamics of the emulated system (1)-(2), shown in Fig. 1 (a), by a hybrid experiment with bidirectional real-time coupling as in Fig. 1 (b). Any trajectory of the emulated system (1)-(2) will eventually spend some time $\delta$ near $\theta=0$ or $\pi$. The time $\delta$ is considerably larger than the delay $\tau$, which is approximately $10 \mathrm{~ms}$. During this time the essential instability of the linearised time map amplifies any small disturbances within the hybrid experiment to order 1. 


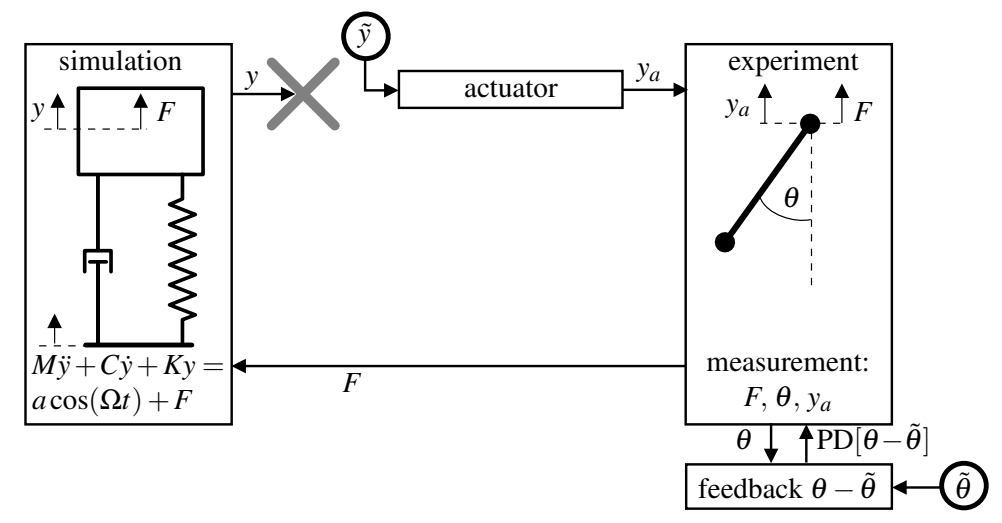

Fig. 2. Sketch of the iterative hybrid experiment. The periodic input $\tilde{y}$ replaces the coupling from the simulation to the experiment. Additionally, a classical (proportional-plus-derivative) feedback loop stabilises the parametrically driven pendulum. The system has two additional periodic inputs: $\tilde{\theta}, \tilde{y}$ (in black circles). A model for this setup is system (3), (22), (23).

\section{Interface matching by Newton iteration}

As we demonstrate now, it is still possible to perform a systematic analysis of the dynamics of the emulated system of Fig. 1(a) by studying the hybrid system. To this end, we break the coupling in one direction, match the output at the interface by a Newton iteration and exploit some fundamental ideas from bifurcation theory.

Fig. 2 shows how the coupling between the two subsystems is relaxed by breaking the bidirectional coupling. Instead, the actuator is fed with a periodic demand $\tilde{y}(t)$ (for example, we choose the period $4 \pi / \Omega$ for $\tilde{y}$ in Section 6 ). After this modification the experimental component is a parametrically (periodically) excited pendulum. The force output $F(t)$ of the experiment still enters the simulation. In addition, we stabilise the parametrically excited pendulum by a classical feedback loop (for example, with proportional-plus-derivative control) with a periodic demand signal $\tilde{\theta}(t)$, which is also of period $4 \pi / \Omega$ :

$$
\operatorname{PD}[\theta-\tilde{\theta}](t)=k_{1}[\theta(t)-\tilde{\theta}(t)]+k_{2}[\dot{\theta}(t)-\dot{\tilde{\theta}}(t)] .
$$

In general, a small delay in the control loop does not impede the ability of classical feedback to stabilise the experimental part. Our approach is general in the sense that it can be applied whenever one can implement a stabilising feedback control loop. How one can implement this loop depends on the particular experimental setup. We discuss some possibilities for the implementation of the control loop and comment on the choice of control gains $k_{1}$ and $k_{2}$ for the pendulum-MSD system in Section 6 .

With the two modifications shown in Fig. 2 the hybrid system is only uni- 
directionally coupled and feedback-stabilised. For any pair of inputs $(\tilde{\theta}, \tilde{y})$ of period $4 \pi / \Omega$ the outputs $\theta(t), y(t)$ and $y_{a}(t)$ of this system have also period $4 \pi / \Omega$ after a short transient. Moreover, the output $\left(\theta, y, y_{a}\right)$ depends smoothly on the input $(\tilde{\theta}, \tilde{y})$ and all parameters. In general the output $\left(\theta, y, y_{a}\right)$ (and the overall dynamics) of the system shown in Fig. 2 will be different from the dynamics of the original emulated system shown in Fig. 1(a). However, if we find an input $(\tilde{\theta}, \tilde{y})$ of period $4 \pi / \Omega$ such that the output satisfies for all times the conditions

$$
\begin{aligned}
& 0=\theta(t)-\tilde{\theta}(t), \\
& 0=y_{a}(t)-y(t),
\end{aligned}
$$

then the trajectory of the partially decoupled and stabilised system in Fig. 2 is identical to an oscillation of period $4 \pi / \Omega$ of the original emulated system in Fig. 1(a). Condition (17) implies that the actual control effort is zero, that is, the feedback control is non-invasive. Condition (18) guarantees that the synchronisation error is zero. The right-hand-sides of (17) and (18) are two periodic functions that depend nonlinearly but smoothly on the two periodic inputs $\tilde{\theta}$ and $\tilde{y}$ and on all system parameters.

As a consequence, it is possible to employ a Newton iteration to find inputs $\tilde{\theta}$ and $\tilde{y}$ satisfying the conditions (17) and (18). Newton iteration finds roots by solving systems of equations of the form

$$
\mathbf{0}=\mathbf{F}(\mathbf{z})
$$

for a smooth vector-valued nonlinear function $\mathbf{F}$ (the residual) and a vector $\mathbf{z}$ of the same dimension by applying the iteration

$$
\mathbf{z}_{l+1}=\mathbf{z}_{l}-\left(\mathbf{F}_{l}^{\prime}\right)^{-1} \mathbf{F}\left(\mathbf{z}_{l}\right),
$$

where $\mathbf{F}_{l}^{\prime}$ is an approximation of the Jacobian of $\mathbf{F}$ in $\mathbf{z}_{l}$. In our case the variable $\mathbf{z}$ is the periodic vector function $(\tilde{\theta}, \tilde{y})$ and the residual $\mathbf{F}$ is the righthand-side of (17)-(18), which is also periodic. In practice, we project $(\tilde{\theta}, \tilde{y})$ and the residual onto the leading $m$ Fourier modes to obtain a finite-dimensional vector $\mathbf{z}$ and residual $\mathbf{F}$. If the function $\mathbf{F}$ is not analytically known, as is the case in our method, then the matrix $\mathbf{F}_{l}^{\prime}$ has to be obtained either by a finite difference approximation or by a recursion. We use here the Broyden rank-1 update [23] given by

$$
\mathbf{F}_{l+1}^{\prime}=\mathbf{F}_{l}^{\prime}+\frac{\left(\mathbf{F}\left(\mathbf{z}_{l}\right)-\mathbf{F}\left(\mathbf{z}_{l-1}\right)\right)-\mathbf{F}_{l}^{\prime}\left[\mathbf{z}_{l}-\mathbf{z}_{l-1}\right]}{\left(\mathbf{z}_{l}-\mathbf{z}_{l-1}\right)^{T}\left(\mathbf{z}_{l}-\mathbf{z}_{l-1}\right)}\left(\mathbf{z}_{l}-\mathbf{z}_{l-1}\right)^{T} .
$$

Thus, we can apply the Newton iteration even if we do not know an analytical expression for $\mathbf{F}$. All that is required is a good initial guess $\mathbf{z}_{0}$ and the ability to evaluate the residual $\mathbf{F}$ at a sequence of points $\mathbf{z}_{l}$ given by the recursion (20). 
One evaluation of $\mathbf{F}$ in a point $\mathbf{z}=(\tilde{\theta}, \tilde{y})$ involves the following procedure.

(1) Set the periodic function $\tilde{\theta}(t)$ as the control target in the feedback control (16) and the periodic function $\tilde{y}(t)$ as the actuator input. Both have period $4 \pi / \Omega$.

(2) Wait until the experiment has settled to a periodic output of period $4 \pi / \Omega$ and measure the (periodic) outputs $\theta(t), y(t)$ and $y_{a}(t)$ over one period. Use these outputs to evaluate the right-hand-sides of (17)-(18).

(3) Project the right-hand-sides of (17)-(18) onto the first $m$ Fourier modes to obtain $\mathbf{F}$.

This iterative procedure does not require any knowledge of the underlying model for the experimental component of the hybrid system in Fig. 2, nor do we need a model for the dependence of the actuator output $y_{a}$ on the force $F$ and the prescribed trajectory $\tilde{y}$ (say, (5) or (11)).

The problem of finding a good initial guess $\mathbf{z}_{0}$ can be solved by embedding the Newton iteration into a path-following procedure as described in $[2,12]$. We extend $\mathbf{z}$ by a system parameter (for example, $\mathbf{z}=(\tilde{\theta}, \tilde{y}, a)$ ). The solutions of (19) form a smooth curve that corresponds to a one-parameter family of periodic motions of the original emulated system in Fig. 1(a). One can trace out this solution curve by extending the nonlinear system (19) with the pseudoarclength condition

$$
\mathbf{z}_{\text {tan }}^{T}\left[\mathbf{z}-\mathbf{z}_{\text {old }}\right]=s .
$$

Here $\mathbf{z}_{\text {old }}$ is the previous point on the curve, $\mathbf{z}_{\tan }$ is an approximation to the tangent at the curve in $\mathbf{z}_{\text {old }}\left(\mathbf{z}_{\tan }\right.$ can be any unit vector that is not orthogonal to the curve), and $s$ is a small parameter determining the step size along the curve. The solution of (19) extended by (21) gives the next point along the curve. A good initial guess for the Newton iteration is $\mathbf{z}_{0}=\mathbf{z}_{\text {old }}+s \mathbf{z}_{\text {tan }}$. How one can find a good starting point for the curve depends strongly on the specific system (see Section 6 for the pendulum-MSD example). The embedding into a one-parameter family guarantees that the Newton iteration converges for small $s$ as long as the solution curve is regular [12]. It also guarantees that the transients of the experimental evaluation of the residual $\mathbf{F}$ are short and that the method can be applied even if the feedback control achieves only local stabilisation.

\section{A case study of periodic orbit continuation}

In this section we demonstrate with the example of the hybrid pendulum-MSD system how our approach can be used to investigate the dynamics of the emulated system even for the case $m>M$. First, we determine for which values of the forcing frequency $\Omega$ and the amplitude $a$ the hanging-down solution $\theta=0$ 
is dynamically stable by tracking its period doubling bifurcation as a curve in the $(\Omega, a)$-plane. Second, we follow period-doubled oscillations and consider their stability properties.

From the experimental point of view, the only requirement for the feasibility of our approach is the ability to introduce a stabilising feedback control loop into the experimental part. The nature of this control depends strongly on the specific experiment and even on the specific dynamical regime one wants to study. For the period doubling of $\theta=0$ in the hybrid pendulum-MSD system this means that we need to ensure that the feedback stabilises the hangingdown state $\theta=0$ of the parametrically excited pendulum. This cannot be achieved by simply superimposing control onto the vertical periodic excitation. One possibility is the addition of a torque control actuator to the experiment. However, this requires a physical change of the experimental setup, which is why we restrict ourselves to a proof-of-concept simulation study in this section.

Specifically, the experimental part of the system is evaluated by a separate computer simulation of

$$
\ddot{\theta}+\frac{1}{l} \sin \theta \ddot{y}_{a}+\frac{\kappa}{m l^{2}} \dot{\theta}+\frac{g}{l} \sin \theta=-\operatorname{PD}[\theta-\tilde{\theta}](t-\tau),
$$

which corresponds to the application of torque on the pivot of the pendulum to achieve stabilising feedback control. We choose the control gains in the PD control as $k_{1}=4 \mathrm{~N} / \mathrm{m}, k_{2}=4 \mathrm{~kg} / \mathrm{s}$ and assume for our simulation that the actuator is described by the actuator model (5) where the delay is set to $\tau=10 \mathrm{~ms}$. The measured force in (3) is modelled as

$$
F(t)=-m \ddot{y}_{a}-m l\left[\ddot{\theta} \sin \theta+\dot{\theta}^{2} \cos \theta\right] .
$$

We also take into account the limited accuracy that would be present for an experiment. Namely, we use only three relevant digits of the evaluations of the residual in (17)-(18).

Period doubling of hanging-down state In the original emulated system (1)-(2) the hanging-down state $\theta=0$ loses its dynamical stability in a period doubling bifurcation. Standard bifurcation theory states that near this loss of stability a solution of period $4 \pi / \Omega$ with a small harmonic amplitude of the angle $\theta$ emerges [2]. Hence, in order to find the boundary of stability of the hanging-down state it is sufficient to track small period-two solutions.

We choose the vector of variables $\mathbf{z}=\left(\tilde{\theta}_{1}, \tilde{y}_{1}, a, \Omega\right)$ where $\tilde{\theta}_{1}(t)=\tilde{\theta}(\Omega t /(4 \pi))$, $\tilde{y}_{1}(t)=\tilde{y}(\Omega t /(4 \pi))$ (thus, scaling the periodic components of $\mathbf{z}$ to period 1 ). As $\mathbf{z}$ has one additional component $\Omega$ we append (19),(21) by the bifurcation 


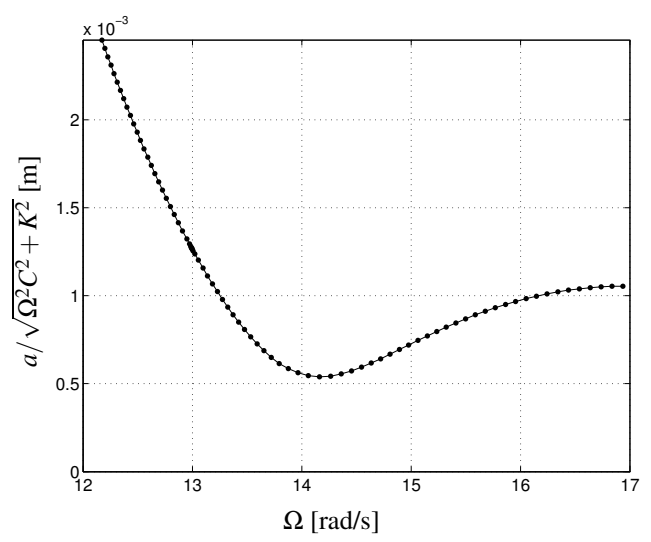

Fig. 3. Continuation of the period-doubling yields a stability curve in the twoparameter plane of $\Omega$ vs. the amplitude $a / \sqrt{\Omega^{2} C^{2}+K^{2}}$. Dots along the curve mark the steps of the continuation. Parameters of the simulated subsystem are $C=0.1 \mathrm{~kg} / \mathrm{s}, K=200 \mathrm{~N} / \mathrm{m}, M=0.2 \mathrm{~kg}$; parameters of the pendulum are $m=0.27 \mathrm{~kg}, l=0.1955 \mathrm{~m} \kappa=7.5 \cdot 10^{-3} \mathrm{~kg} / \mathrm{s}$, delay $\tau=10^{-2} \mathrm{~s}$; control gains are $k_{1}=4 \mathrm{~N} / \mathrm{m}, k_{2}=4 \mathrm{~kg} / \mathrm{s}$; the amplitude of small angular oscillation is $r=0.1 \mathrm{rad}$.

condition

$$
\int_{0}^{1}\left[\tilde{\theta}_{1}(t)\right]^{2} \mathrm{~d} t=r^{2}
$$

for a small fixed $r$ (which fixes the amplitude of the angle $\theta$ of the period-two solutions). The system (19),(21),(24) defines a sequence of points along the approximate (because $r>0$ ) period-doubling bifurcation curve in which the hanging-down state $\theta=0$ of the emulated system (1)-(2) loses its stability. Since (21) and (24) are known analytically, we also know the corresponding rows of the Jacobian $\mathbf{F}^{\prime}$ in (19) analytically. We approximate the two periodic functions $\tilde{\theta}_{1}$ and $\tilde{y}_{1}$ by their first two Fourier modes

$$
\begin{aligned}
& \tilde{y}_{1}(t)=y_{0}+y_{1} \exp (2 \pi i t)+y_{2} \exp (4 \pi i t), \\
& \tilde{\theta}_{1}(t)=\theta_{0}+\theta_{1} \exp (2 \pi i t)+\theta_{2} \exp (4 \pi i t),
\end{aligned}
$$

where $y_{0}, \theta_{0} \in \mathbb{R}, y_{1}, y_{2}, \theta_{1}, \theta_{2} \in \mathbb{C}$. After a Galerkin projection onto these two Fourier modes, (17)-(18) together with (24), form a system of eleven (realvalued) equations for twelve (real-valued) variables. It implicitly defines a curve that can be traced by the path-following method described in Section 5 . The curve of period-doublings thus obtained is shown in Fig. 3.

Since $r$ is not zero but small and positive the computed curve is shifted slightly into the region of stability of the hanging-down state whenever the emerging $4 \pi / \Omega$-periodic solutions are unstable (subcritical period doubling). Similarly, it is shifted slightly into the region of instability whenever the emerging $4 \pi / \Omega$ periodic solutions are stable (supercritical period doubling). The size of the shift is of the order $O\left(r^{2}\right)$. 
(a)
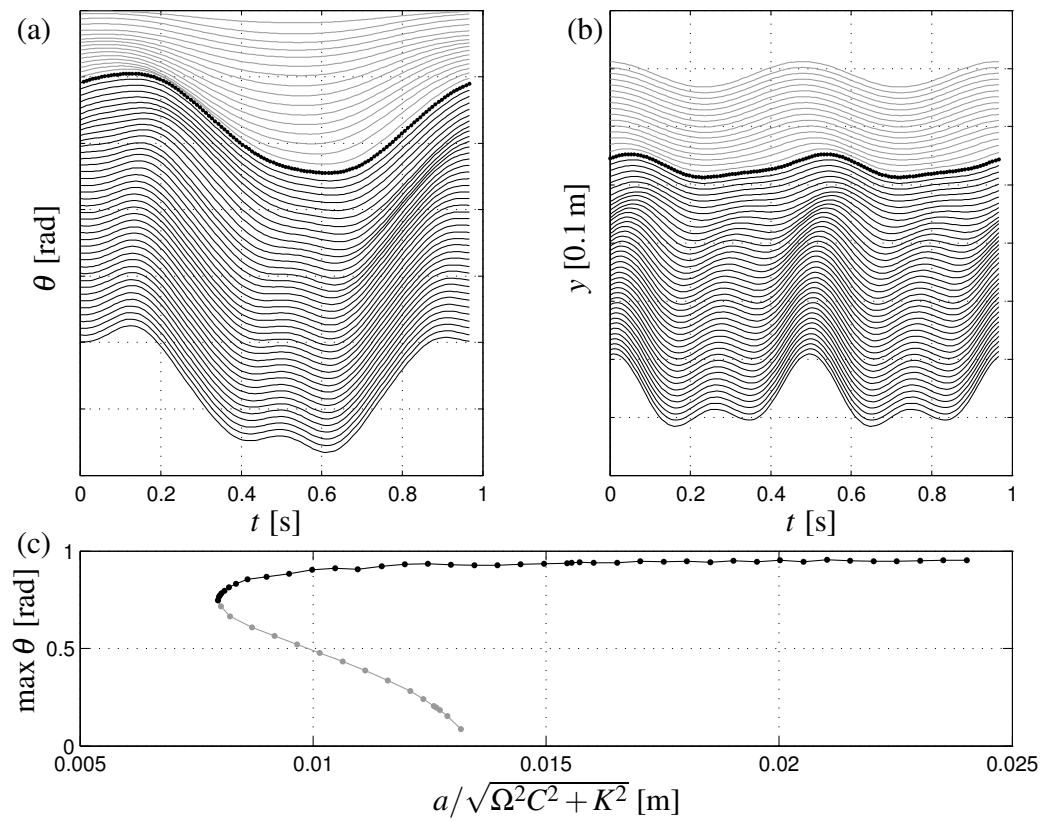

Fig. 4. One-parameter continuation for $\Omega=13 \mathrm{rad} / \mathrm{s}$. Shown are time profiles of $\tilde{\theta}$ (a) and of $\tilde{y}$ (b) in a waterfall plot, and the one-parameter bifurcation diagram (c). Time profiles corresponding to unstable periodic orbits are grey, and time profiles corresponding stable periodic orbits are black; the saddle-node orbit in between the two cases, which corresponds to the fold point in panel (c), is shown as thick line. The unit in the label of the vertical axis in the panels (a) and (b) corresponds to the distance between horizontal grid lines.

Symmetric periodic orbits Fig. 4 shows the one-parameter family of symmetric $4 \pi / \Omega$-periodic orbits branching off from the period doubling curve for fixed $\Omega=13 \mathrm{rad} / \mathrm{s}$. The family has been computed with the path-following approach described in Section 5, starting from the solution with zero amplitude of $\tilde{\theta}$. The periodic functions $\tilde{\theta}_{1}$ and $\tilde{y}_{1}$ are discretised by six (complex) Fourier modes. Panel (c) shows the maximum of $\tilde{\theta}$ along the periodic orbit depending on the parameter $a$ in a one-parameter bifurcation diagram. The family emerges 'backwards' from the period doubling (at $0.013 \mathrm{~m}$ ), which shows that the period doubling is subcritical. The family is unstable initially until it folds back at a saddle-node bifurcation (at $0.008 \mathrm{~m}$ ) where it becomes stable. Pathfollowing is able to find the periodic orbits independently of their dynamical stability in the original emulated system (1)-(2). In fact, the dynamical stability of the orbit is not detected along the curve; it was concluded from the position of the orbit in the bifurcation diagram, relative to the fold point. Fig. 4 (a) and (b) show the time profiles of $\tilde{\theta}$ and $\tilde{y}$ (on the interval $[0,4 \pi / \Omega]$ ) in a waterfall plot. The grey profiles correspond to the unstable part of the family, the black profiles lie on the stable part. The profile of the saddle-node orbit is highlighted as a thick line. It separates the stable and unstable parts of the branch. 

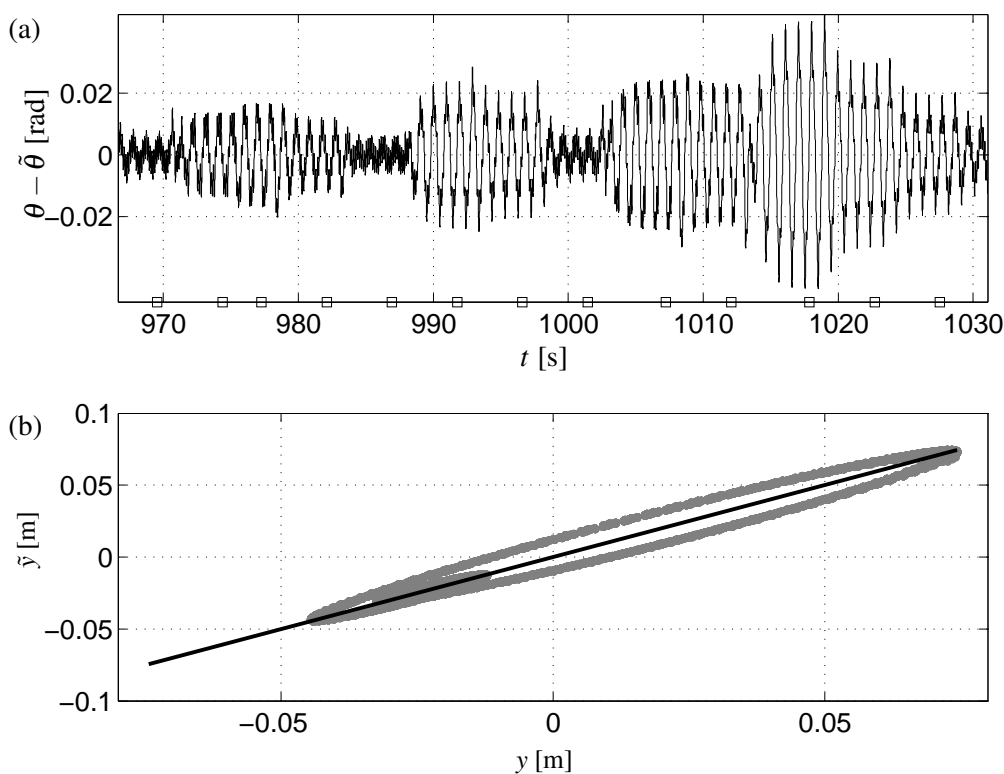

Fig. 5. Illustration of the Quasi-Newton iteration during path-following. Panel (a) shows the time profile of the Quasi-Newton iteration (along the branch shown in Fig. 4(c) for a forcing parameter near 0.02), that is, successively changing inputs until the control input $\theta-\tilde{\theta}$ becomes small. The small squares at the bottom of panel (a) indicate when the outputs $\theta, y$ and $y_{a}$ have settled to a periodic motion. Panel (b) shows the mismatch between actuator input $\tilde{y}$ and output $y_{a}$.

Fig. 5 (a) shows a typical time profile of the feedback control input $\theta-\tilde{\theta}$ during the path-following along the curve in Fig. 4(c). Note that the transients, occurring whenever $\tilde{\theta}, \tilde{y}$ and $a$ are changed, are typically small because these inputs are varied only gradually during the continuation. The squares along the time axis indicate when the system is considered to have settled down to a periodic state. At these time points the last period of the outputs $\theta, y$, and $y_{a}$ is recorded for the evaluation of the right-hand-sides of (18) and (17), and new inputs and parameters are set. Fig. 5 (b) shows the offset between $y$ and $\tilde{y}$ in the $(y, \tilde{y})$-plane. This offset highlights how much $\tilde{y}$ anticipates the output $y$ of the simulation. Importantly, we achieve synchronisation without expressly exploiting the knowledge about the actuator model (5).

\section{Conclusion and outlook}

We demonstrated with a simulation study of a substructured pendulum-MSD system that control-based bifurcation analysis is feasible even for hybrid systems suffering from a delay-induced essential instability. The proposed method does not rely on a particular model for the transfer system connecting the computer simulation and the experiment. It is able to deal with arbitrary unknown 
actuator dynamics, most notably, it compensates the effect of delay in the actuation. This is in contrast to classic delay compensation schemes, which are not able to overcome an essential instability. The incorporation of controlbased bifurcation analysis into the hybrid experiment of the pendulum-MSD system itself is currently in preparation.

As is explained in [8] our approach is general: it allows for systematic bifurcation analysis in experiments that have a stabilising feedback loop. The underlying idea is to relax the coupling between the two components, so that the experimental subsystem is periodically driven and feedback stabilised with a suitable control input. The condition that the control target be equal to the actual output of the experimental subsystem can be solved iteratively by Newton iteration. One function evaluation corresponds in this context to running the experiment until the system has settled down in response to the prescribed input. Non-invasive control and perfect synchronisation correspond to the reproduction of the dynamics of the emulated system. The iterative nature of our method removes the real-time constraint for the numerical component of the overall hybrid test. Therefore, control-based bifurcation analysis may be particularly useful in situations when the numerical component is so complex that its evaluation is too time-consuming to satisfy real-time constraints.

Even though our method is iterative with relaxed real-time constraints, it is still fully dynamic. This is an important difference with pseudo-dynamic substructured tests as they are performed for very large structures, for example, in earthquake engineering. For the sheer impossibility of real-time displacement computation and transfer, such tests are run iteratively with quasi-static loading; see [24] for an example involving a bridge pier. Therefore, dynamic forces must be estimated, which makes it very difficult to capture instabilities of the original emulated system with a pseudo-dynamic test. By contrast, in our approach no force estimates are necessary. Indeed, the dynamics that is recorded when the system is synchronised and the control action is zero is exactly that (within the measurement errors) of the emulated system. As we have shown with the example of period doubling in the pendulum-MSD system, this allows one to track stability boundaries directly in system parameters.

Apart from the experimental validation of our proposed methodology, there are a number of other challenges for future research. We mention here the incorporation of other bifurcations, such as the saddle-node bifurcation or torus bifurcation (some bifurcations have been studied in [8]), the detection of bifurcations along families of periodic solutions, and the development of methods for strongly nonlinear phenomena (such as homoclinic orbits). 


\section{Acknowledgements}

We thank Alicia Gonzalez-Buelga, Yuliya Kyrychko, Simon Neild and David Wagg for helpful discussion on hybrid testing and the pendulum-MSD experiment.

\section{References}

[1] J. Guckenheimer, P. Holmes, Nonlinear Oscillations, Dynamical Systems, and Bifurcations of Vector Fields, Vol. 42 of Applied Mathematical Sciences, Springer-Verlag, New York, 1983, re-issued 1990.

[2] Y. A. Kuznetsov, Elements of Applied Bifurcation Theory, 3rd Edition, Vol. 112 of Applied Mathematical Sciences, Springer-Verlag, New York, 2004.

[3] W. Govaerts, Y. Kuznetsov, Interactive continuation tools, in: B. Krauskopf, H. Osinga, J. Galán-Vioque (Eds.), Numerical Continuation Methods for Dynamical Systems: Path Following and Boundary Value Problems, SpringerVerlag, Dordrecht, 2007, pp. 51-75.

[4] A. Blakeborough, M. Williams, A. Darby, D. Williams, The development of real-time substructure testing, Philosophical Transactions of the Royal Society of London A 359 (2001) 1869-1891.

[5] M. Wallace, D. Wagg, S. Neild, An adaptive polynomial based forward prediction algorithm for multi-actuator real-time dynamic substructuring, Proceedings of the Royal Society of London A 461 (2005) 3807-3826.

[6] Y. Kyrychko, K. Blyuss, A. Gonzalez-Buelga, S. Hogan, D. Wagg, Real-time dynamic substructuring in a coupled oscillator-pendulum system, Proceedings of the Royal Society of London A 462 (2006) 1271-1294.

[7] A. Gonzalez-Buelga, D. Wagg, S. Neild, Parametric variation of a coupled pendulum-oscillator system using real-time dynamic substructuring, Structural Control and Health Monitoring 14 (7) (2007) 991-1012.

[8] J. Sieber, B. Krauskopf, Control based bifurcation analysis for experiments, Nonlinear Dynamics (in press), online first at http://dx.doi.org/10.1007/ s11071-007-9217-2.

[9] J. Sieber, B. Krauskopf, Control-based continuation of periodic orbits with a time-delayed difference scheme, International Journal of Bifurcation and Chaos 17 (8) (2007) 2579-2593.

[10] M. Nakashima, Development, potential and limitations of real-time online (pseudo dynamic) testing, Philosophical Transactions of the Royal Society A 359 (2001) 1851-1867. 
[11] E. J. Doedel, A. R. Champneys, T. F. Fairgrieve, Y. A. Kuznetsov, B. Sandstede, X. Wang, AUTO97, Continuation and bifurcation software for ordinary differential equations, Concordia University (1998).

[12] E. Doedel, Lecture notes on numerical analysis of nonlinear equations, in: B. Krauskopf, H. Osinga, J. Galán-Vioque (Eds.), Numerical Continuation Methods for Dynamical Systems: Path Following and Boundary Value Problems, Springer-Verlag, Dordrecht, 2007, pp. 51-75.

[13] M. Wallace, J. Sieber, S. Neild, D. Wagg, B. Krauskopf, Stability analysis of real-time dynamic substructuring using delay differential equation models, International Journal of Earthquake Engineering and Structural Dynamics 34 (15) (2005) 1817-1832.

[14] T. Horiuchi, T. Konno, A new method for compensating actuator delay in realtime hybrid experiments, Philosophical Transactions of the Royal Society A 359 (2001) 1893-1909.

[15] A. Darby, A. Blakeborough, M. Williams, Improved control algorithm for realtime substructure testing, International Journal of Earthquake Engineering and Structural Dynamics 30 (2001) 431-448.

[16] A. Darby, M. Williams, A. Blakeborough, Stability and delay compensation for real-time substructure testing, Journal of Engineering Mechanics 128 (2002) $1276-1284$.

[17] J. K. Hale, S. M. Verduyn Lunel, Introduction to Functional-Differential Equations, Vol. 99 of Applied Mathematical Sciences, Springer-Verlag, New York, 1993.

[18] O. Diekmann, S. A. van Gils, S. M. Verduyn Lunel, H.-O. Walther, Delay Equations, Vol. 110 of Applied Mathematical Sciences, Springer-Verlag, New York, 1995, functional, complex, and nonlinear analysis.

[19] A. Pazy, Semigroups of Linear Operators and Applications to Partial Differential Equations, Vol. 44 of Applied Mathematical Sciences, SpringerVerlag, New York, 1983.

[20] Q. Wang, T. Lee, K. Tan, Finite Spectrum Assignment for Time-Delay Systems, Vol. 239 of Lecture Notes in Control and Information Sciences, Springer-Verlag, New York, 1999.

[21] T. Insperger, Act-and-wait concept for continuous-time control systems with feedback delay, IEEE Transactions on Control Systems Technology 14 (5) (2006) 974-977.

[22] J. Sieber, Dynamics of delayed relay systems, Nonlinearity 19 (11) (2006) 24892527.

[23] V. Eyert, A comparative study on methods for convergence acceleration of iterative vector sequences, Journal of Computational Physics 124 (0059) (1996) $271-285$. 
[24] A.V. Pinto, P. Pegon, G. Magonette, G.Tsioni, Pseudo-dynamic testing of bridges using non-linear substructuring Earthquake Engineering ans Structural Dynamics 33 (11) (2004) 1125-1146.

\section{A Reduction of the delay equation model to its essential part}

Following $[17,18]$ we rewrite (6) as an explicit first-order system, where we denote the components by $(u, \theta, y, v, w)$ : namely, $u([t-\tau, t])$ is the history segment of $\dot{y}, v$ is an auxiliary scalar variable, and $w$ is a modification of $\dot{\theta}$. The evolution of $(u, \theta, y, v, w)$ that is equivalent to (6) is given by

$$
\begin{aligned}
u(t) & =\left[-\frac{m}{M} \cos ^{2} \theta(t)\right] u(t-\tau)+\frac{1}{M} v(t), \\
\dot{y}(t) & =u(t), \\
\dot{\theta}(t) & =w(t)-\frac{\sin \theta(t)}{l}, \\
\dot{v}(t) & =f_{1}(t, u(t), u(t-\tau), \theta(t), y(t), w(t)), \\
\dot{w}(t) & =f_{2}(u(t-\tau), \theta(t), w(t)),
\end{aligned}
$$

where the functions $f_{1}$ and $f_{2}$ are

$$
\begin{aligned}
f_{1}\left(t, u_{0}, u_{1}, \theta, y, w\right)= & \frac{\kappa}{l^{2}}[l w-\sin \theta] \sin \theta+m g \sin ^{2} \theta-C u_{0}-K y+a \cos (\Omega t) \\
& -\frac{m}{l}[l w-\sin \theta]^{2} \cos \theta-\frac{m}{l}[l w-\sin \theta] \sin (2 \theta) u_{1}, \\
f_{2}\left(u_{1}, \theta, w\right)= & \frac{1}{l^{2}}\left[\cos \theta u_{1}-\frac{\kappa}{m}\right][l w-\sin \theta]-\frac{g}{l} \sin \theta .
\end{aligned}
$$

The delayed value $u(t-\tau)$ of $u$ enters the functions $f_{1}$ and $f_{2}$ in the argument $u_{1}$. The set of equations (A.1)-(A.5) gives a well-defined dynamical system for $(u, y, \theta, v, w)$. The solution of this set of equations on an interval $\left(t_{1}, t_{1}+\tau\right]$ is uniquely defined by the variation-of-constants formulation of (A.1)-(A.5); see [19].

The appropriate initial condition $\mathbf{x}$ (over the delay interval) consists of one function segment $u\left(\left[t_{1}-\tau, t_{1}\right]\right)$ and the four scalars $y\left(t_{1}\right), \theta\left(t_{1}\right), v\left(t_{1}\right)$ and $w\left(t_{1}\right)$. Note that the trajectory of $u$ is only continuous in $t_{1}$ if (A.1) is satisfied for the initial condition $\mathbf{x}$. If we require continuity for $u$ this effectively gives a nonlinear condition on the initial condition of the auxiliary variable $v$. Only such continuous velocities are physically relevant, but note that system (A.1)(A.5) can also be solved for square-integrable functions $u$. 
The theory for systems composed of difference equations and ODEs is well developed; see, for example, the text books $[17,18]$. It is known that the time map $\mathbf{X}\left(t_{2}, t_{1} ; \mathbf{x}\right)$, given by the evolution of (A.1)-(A.5) from time $t_{1}$ to time $t_{2}$ starting from the initial condition $\mathbf{x}$, is smooth with respect to its argument $\mathbf{x}$ (the initial condition) whenever the right-hand-sides are smooth functions. We denote the derivative of $\mathbf{X}$ with respect to its third argument (the initial condition $\mathbf{x}$ ) by $\partial_{3} \mathbf{X}$. The derivative $\partial_{3} \mathbf{X}$ can be computed by solving the variational equations for (A.1)-(A.5).

To show that for $m>M$ the time map $\mathbf{X}$ depends extremely sensitively on its initial condition $\mathbf{x}$ when passing through the hanging-down position $\theta=0$ we have to compute the spectrum of the linear map $\partial_{3} \mathbf{X}\left(t_{1}+\delta, t_{1} ; \hat{\mathbf{x}}_{*}\right)$ for an initial condition $\hat{\mathbf{x}}_{*}$ which corresponds to the hanging-down position of the pendulum. Let us denote the trajectory of $\mathbf{X}$ on the interval $\left[t_{1}, t_{1}+\delta\right]$ starting from $\hat{\mathbf{x}}_{*}$ by

$$
\left.\mathbf{x}_{*}(t)=\left[u_{*}(t), \theta_{*}(t), y_{*}(t), v_{*}(t), w_{*}(t)\right] \text { for } t \in\left[t_{1}, t_{1}+\delta\right]\right),
$$

where $\theta_{*}\left(t_{1}\right)=0$ and $\mathbf{x}_{*}\left(t_{1}\right)=\hat{\mathbf{x}}_{*}$. We denote the solution of the variational equations for system (A.1)-(A.5) in $\mathbf{x}_{*}(t)$ on the time interval $\left[t_{1}, t_{1}+\delta\right]$ by

$$
\mathbf{x}(t)=[u(t), \theta(t), y(t), v(t), w(t)] .
$$

The variational equation of the difference equation (A.1) is

$$
u(t)=\left[-\frac{m}{M} \cos ^{2} \theta_{*}(t)\right] u(t-\tau)+\frac{1}{M} v(t)+\left[\frac{m}{M} \sin \left(2 \theta_{*}(t)\right) u_{*}(t-\tau)\right] \theta(t),
$$

while the variational equations for $\theta, y, v$ and $w$ are linear ODEs depending on $u(t)$ and $u(t-\tau)$. Textbook [17] explains that the essential spectral radius of the time map $\partial_{3} \mathbf{X}\left(t_{1}+\delta, t_{1} ; \hat{\mathbf{x}}_{*}\right)$ is determined completely by the difference equation part (A.6) of the variational equation. The other variables do not play a role, which means we can drop the terms containing $v$ and $\theta$ from (A.6). Thus, the essential spectral radius of $\partial_{3} \mathbf{X}\left(t_{1}+\delta, t_{1} ; \hat{\mathbf{x}}_{*}\right)$ is the same as the essential spectral radius $\rho$ of the difference equation that appears as Equation (8) in Section 3. Moreover, according to [17], $\partial_{3} \mathbf{X}\left(t_{1}+\delta, t_{1} ; \hat{\mathbf{x}}_{*}\right)$ has countably infinitely many eigenvalues approaching this essential spectral radius $\rho$. 